BOmmun:HEIT! Communication et organisation

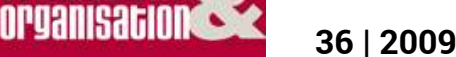

Pour une approche communicationnelle de l'individu au travail

\title{
Visibilité et reconnaissance de l'individu au travail
}

Yanita Andonova et Béatrice Vacher

\section{(2) OpenEdition}

Journals

Édition électronique

URL : http://journals.openedition.org/communicationorganisation/970

DOI : 10.4000/communicationorganisation.970

ISSN : $1775-3546$

Éditeur

Presses universitaires de Bordeaux

Édition imprimée

Date de publication : 1 décembre 2009

Pagination : 136-147

ISBN : 978-2-86781-719-9

ISSN : 1168-5549

Référence électronique

Yanita Andonova et Béatrice Vacher, "Visibilité et reconnaissance de l'individu au travail ",

Communication et organisation [En ligne], 36 | 2009, mis en ligne le 11 mars 2011, consulté le 19 avril

2019. URL : http://journals.openedition.org/communicationorganisation/970 ; DOI : 10.4000/

communicationorganisation.970 
Dossier : Pour une approche communicationnelle de l'individu au travail

\title{
Résumé
}

Les théories de la reconnaissance revisitées dans une approche communicationnelle sont au cœur de la réflexion proposée. En confrontant deux terrains organisationnels qui partent de la même question de l'invisibilité des TIC, l'étude aboutit à des conclusions opposées. La reconnaissance de l'individu au travail, processus communicationnel d'estime mutuelle, émerge dans l'interaction, se manifeste de part et d'autre par de la gratitude et interpelle la question centrale de la visibilité des actions.

\section{Mots clefs}

Reconnaissance, TIC, approche communicationnelle, visibilité

\begin{abstract}
The authors revisit the theory of recognition in a communicative approach by comparing two organizational fields, which spring from the same issue of the invisibility of ICT. This study leads to radically different conclusions. The recognition of the individual at work - a communicative process of mutual esteem - emerges in the interaction and occurs on both sides by the gratitude and challenges the central issue of the actions visibility.
\end{abstract}

Keywords

Recognition, ICT, communicative approach, visibility

Yanita Andonova est docteur en communication du CELSA (Université Paris IV Sorbonne), ingénieur au Conservatoire National des Arts et Métiers (Cnam) et chercheur dans l'équipe DICEN.

Béatrice Va cher est chercheur associée au laboratoire CHERPA, Institut d'Etudes Politiques d'Aix-en-Provence et membre du conseil d'administration de la Société Française en Sciences de l'Information et de la Communication (SFSIC). 


\title{
Visibilité et reconnaissance de l'individu au travail Yanita Andonova, Béatrice Vacher
}

\author{
yanita.andonova@gmail.com \\ beatricevacher@gmail.com
}

La question de la reconnaissance de l'individu se pose aujourd'hui avec acuité dans l'espace public, la sphère privée et l'univers du travail. Elle devient peu à peu un thème de réflexion pour les organisations face à l'élargissement de leur champ de responsabilité, aux aléas de l'environnement et aux conflits sociaux. La reconnaissance de l'individu au travail se pose à la fois en termes de revendications identitaires (respect des identités professionnelles, reconnaissance des métiers), de motivation professionnelle (gestion des compétences, valorisation sociale) mais aussi de légitimité institutionnelle (place et statut plus ou moins cohérents par rapport aux rôles effectifs).

La réflexion engagée ici part du postulat suivant : la reconnaissance de l'individu au travail est un processus communicationnel mutuel mais pas nécessairement réciproque. En effet, la reconnaissance émerge dans l'interaction (équipée) mais elle n'est pas un simple échange, elle est un partage. Elle se manifeste de part et d'autre par de la gratitude qui prend toutefois des formes différentes (sourire, gratification pécuniaire, honneurs, etc.) et dépend des places respectives de chacun (subordonné, responsable, directeur) ainsi que des situations particulières dans lesquelles elle s'exprime. Adopter une approche communicationnelle de la théorie de la reconnaissance éclaire les expériences quotidiennes de visibilité des tâches selon qui les exécute et qui les juge, ce que nous précisons dans la première partie.

Dans la deuxième partie, nous confrontons deux terrains organisationnels ${ }^{59}$ qui, à partir des tâches professionnelles quotidiennes, nécessitent le recours aux technologies de l'information et de la communication (TIC). Dans les deux cas, ces tâches sont invisibles mais cette invisibilité aboutit à des conséquences radicalement opposées. Comment, au sein des ateliers de production, les TIC valorisent-elles les utilisateurs en quittant la sphère des objets

\footnotetext{
${ }^{59}$ La première étude de cas repose sur une approche inductive de type ethnographique et la seconde sur une recherche action combinant ethnographie de la communication et Grounded Theory (Winkin, 2001 ; Glaser et Strauss, 1967).
} 
Dossier : Pour une approche communicationnelle de l'individu au travail

techniques énigmatiques pour devenir des dispositifs quotidiens invisibles et ainsi, provoquer l'estime de la hiérarchie ? Comment, au contraire, dans un centre de recherche, les TIC dévalorisent-elles les personnes qui en ont la charge en rajoutant d'autres tâches fastidieuses (programmation, classement, tri), dévaluées par la hiérarchie ?

C'est le constat de l'existence de divers registres de reconnaissance que nous souhaitons interroger ici sous l'angle de la visibilité sociale. Ceci nous amène à approfondir en troisième partie les notions d'estime de soi et de valorisation en fonction de la culture dominante contemporaine. Nous verrons alors ce que «font» les dispositifs communicationnels et normatifs comme traducteurs de cette culture autant que comme enjeux de changements de regard mutuel pour les individus au travail.

\section{Approche communicationnelle de la reconnaissance}

En étudiant les actes de communication dans les organisations (langages, discours, pratiques), les dispositifs communicationnels et le sens que les individus attribuent à leurs actions respectives, la communication organisationnelle (d'Almeida, Andonova, 2006) aborde la reconnaissance au travail d'une manière composite. Un certain nombre de travaux démontrent la centralité du langage dans le travail (Lacoste, 2000) et de ses activités d'information et de communication (Guyot, 2004 ; Vacher, 2006) pour en montrer la part structurante et pourtant largement mésestimée. D'autres analysent la compréhension croisée du social et du symbolique en pensant les organisations entre récits et médias (d'Almeida, 2001) et en introduisant dans le champ communicationnel la notion d'interaction pragmatique (Gramaccia, 2001) pour montrer que c'est à ce niveau que se situent les nœuds de reconnaissance. Les liens entre confiance et reconnaissance (Taylor, Cooren, Giroux, Robichaud, 1996), entre communication et représentations sociales (Bouzon, 2002) aident à mieux comprendre l'engagement mutuel au travail (Bernard, Joule, 2004) et renvoient à la question centrale de la visibilité des actions et de la valorisation sociale des individus. Cela se fait sur fond de recomposition des rapports sociaux (Olivesi, 2002), de processus d'inégalités, d'injustice et de domination (Floris, 2006). La reconnaissance au travail que nous proposons d'analyser ici, s'inscrit dans ce mouvement afin de mieux comprendre la transformation du lien social au sein des contextes organisationnels complexes (Boltanski, Chiapello, 1999). Nous précisons la teneur de cette transformation en revisitant les théories de la reconnaissance proposées en sociologie et en philosophie. 
Le sociologue allemand Axel Honneth, directeur de l'École de Francfort, a renouvelé le débat sur la reconnaissance et réintroduit, au sein de l'éthique de la communication, une dimension non prise en compte par Habermas, celle de la «conflictualité sociale » (Habermas, 1987). En partant du déni de reconnaissance et de ses conséquences psychologiques (mépris, humiliation), Honneth $(2000,2006)$ distingue trois sphères de reconnaissance où se constituent de façon complémentaire les formes d'une vie réussie. Dans la sphère de l'intime, la reconnaissance affective est liée aux rapports interpersonnels de proximité, à commencer par les relations familiales et l'amitié. Elle crée un équilibre entre l'état de dépendance et l'autonomie, condition de la confiance en soi (la conscience de son existence en tant qu'être de désirs et de besoins). Dans la sphère publique du droit et du politique, la reconnaissance suppose qu'une personne puisse se sentir porteuse des mêmes droits qu'autrui et développer ainsi un sentiment de respect de soi (la certitude de la valeur de sa liberté). Dans la sphère de la coopération sociale enfin, la solidarité déployée par chacun au sein de la communauté correspond à la reconnaissance accordée à la contribution de celles et ceux qui façonnent la société, et a pour résultat l'estime de soi (la conviction de sa fonction sociale).

Le philosophe français Paul Ricœur insiste quant à lui sur la reconnaissance comme capacité à raconter et recevoir (Ricœur, 2004). Cette reconnaissance commence par soi-même: se reconnaître soimême, c'est se sentir capable de se mettre en intrigue (identité narrative) et de porter les conséquences de ses actes (imputabilité). De là découle la reconnaissance d'autrui qui passe par la gratitude, c'està-dire le «bien recevoir». Recevoir est considéré comme la figure pivot des trois obligations collectives: donner, recevoir et rendre (Mauss, 1925). Ce point de vue rejoint l'approche narrative en communication organisationnelle (Giroux et Marroquin, 2005).

La question de la reconnaissance de l'individu au travail, qui nous intéresse plus particulièrement ici, se situe principalement dans la sphère de la coopération sociale, celle de l'estime de soi. Toutefois, les sphères de la reconnaissance s'articulent et coexistent, le travail étant une dimension constitutive de la vie en société (Méda, 2004). L'estime est fonction de la contribution productive et de l'efficacité des individus, elle s'exprime par le partage de gratitudes (compétences, valeurs) et se décline au travail sous les formes de la coopération, de la responsabilité sociale, de la délégation, etc. Cette précision en appelle alors une autre : pourquoi parler d'individu et non pas de personne ou de sujet? En référence aux théories 
Dossier : Pour une approche communicationnelle de l'individu au travail

interactionnistes, situées et distribuées, nous considérons qu'une personne se constitue dans l'interaction, non seulement humaine mais également équipée symboliquement et matériellement (Suchman, 1987 ; Hutchins, 1995 ; Goffman, 1991 ; Borzeix, Fraenkel, 2005). L'individu est une personne autonome, c'est-à-dire qui a intégré les contraintes (règles, représentations et marquages sociaux) qui la dépassent pour les surmonter et ainsi être capable de les mobiliser (Bouzon, 2002 ; Giroux, 2002). Le sujet serait l'individu émancipé, c'est-à-dire capable de se libérer des contraintes grâce à la méditation pour les transformer en œuvre (Ricœur, 2004 ; Poché, 2004). C'est donc au niveau de l'individu que se situe notre propos.

\section{La reconnaissance à l'épreuve du terrain}

Nous avons choisi de comparer deux terrains organisationnels où les TIC jouent un rôle central et où l'on observe deux conséquences opposées à l'invisibilité grandissante des tâches qui leur sont associées. Cette invisibilité signe, dans le premier cas l'intégration des TIC dans le travail quotidien, ce qui semble favoriser la reconnaissance individuelle, tandis que dans le deuxième cas, elle marque la multiplication des tâches les moins gratifiantes qui ne permettent pas cette reconnaissance. Cette opposition nous semble significative de l'évolution contemporaine des relations de travail.

Le pre mier cas, issu d'une recherche longitudinale par observation participante dans un grand groupe sidérurgique français (Andonova, 2004), concerne l'usage des TIC au sein d'un atelier de production, symbole par excellence de la division du travail et lieu privilégié d'observation des stratégies d'acteurs et des luttes de reconnaissance. Cinq équipes postées, chacune incluant vingt-cinq personnes travaillent $24 \mathrm{~h} / 24$ dans un même espace où estrades d'enfournement, pupitres de commande et ordinateurs s'exposent, coexistent et cohabitent sans empiètement apparent. L'arrivée des TIC dans l'atelier a été l'occasion pour certains de se former, pour d'autres de perfectionner leur utilisation. Mobilisées comme référentiels qualité, bases documentaires, serveur intranet, messagerie électronique, plateforme de formation à distance, valorisées par les discours politique, médiatique et managérial, les TIC sont de plus en plus utilisées dans les ateliers de production et ont conduit, même les plus réticents, à se remettre en question et à faire leurs premiers pas dans l'appropriation de la messagerie et de l'intranet. Petit à petit, l'accès à Internet est ressenti comme un signe d'une plus grande autonomie dans le travail, mais également d'appartenance à «l'avant-garde». Utilisées au quotidien, les TIC finissent par se fondre à leur tour, comme le 
téléphone, le photocopieur et le fax dans le quotidien du travail. C'est seulement alors, lorsqu'elles quittent la sphère des objets techniques énigmatiques, qu'elles apparaissent aux yeux de l'utilisateur comme des dispositifs «ordinaires», «courants» et par conséquent invisibles.

Le désir de reconnaissance des opérateurs par l'institution (le responsable de l'unité de production), par leur responsable hiérarchique direct (le chef de poste) et par leurs collègues d'équipe n'est pas étranger à leur investissement dans l'appropriation des TIC. Les opérateurs tentent sans cesse de justifier la présence de l'ordinateur dans les ateliers et attendent du recours aux TIC un bénéfice hautement gratifiant. L'usage professionnel des TIC est ainsi perçu comme facteur valorisant l'image de l'opérateur, améliorant l'estime de soi. L'invisibilité des TIC, loin d'être une simple standardisation ou un désenchantement de la technique, est l'aboutissement d'un processus souterrain, quotidien, de longue durée, d'adaptation, d'adoption et de «bricolage » de ces dispositifs dans les habitudes professionnelles (De Certeau, 1990, Vacher, 2004). L'appropriation des TIC exige des investissements mutuels : de la part des usagers, une recherche permanente de reconnaissance et de valorisation sociale, de la part de l'entreprise une sensibilisation des salariés aux nouvelles technologies pour faire évoluer leurs habitudes de travail. Dans ce contexte, la transformation du lien social en milieu professionnel passe par l'estime : attente positive de reconnaissance dans et par l'interaction sociale.

La seconde étude est le résultat d'une recherche action de deux années, qui combine ethnographie de la communication et Grounded Theory (Winkin, 2001; Glaser et Strauss, 1967), où le chercheur est également un des protagonistes des situations étudiées. Il a fait l'objet de séminaires de recherche et de croisements théoriques pour produire un terrain susceptible de faire l'objet de comparaisons (Vacher, 2008). La direction d'un centre de recherche a vendu une application intranet haut de gamme, développée en son sein pour favoriser les communautés de pratiques (Giroux, 2006). Le client est jugé important et les bénéfices élevés, ce qui engage une mobilisation des ressources du centre de recherche durant deux années, au détriment d'autres projets. Du point de vue de la direction, le produit vendu est pratiquement fini et ne nécessite que quelques modifications rudimentaires. C'est techniquement le cas. Mais ces évolutions sont nombreuses et demandent une mobilisation complexe de compétences. Les avis sur ce projet divergent donc fortement de la vision de la direction. 
Dossier : Pour une approche communicationnelle de l'individu au travail

Par exemple, un des ingénieurs informaticiens se trouve obligé de délaisser ses projets sophistiqués pour se consacrer durant plusieurs mois à installer des « applets » (petits programmes pour applications Internet) et, de plus, dans l'urgence, ce qu'il déteste. Au lieu de l'encourager, son supérieur hiérarchique lui reproche sa mauvaise volonté. Par ailleurs, un des chercheurs reporte ses travaux en cours pour se consacrer entièrement à la coordination des améliorations du produit. Il s'agit d'articuler les exigences de plusieurs personnes : le directeur du centre de recherche, affirmant que le développement est facile et exigeant que les modifications se fassent rapidement; les utilisateurs, se plaignant des contraintes du produit en le trouvant impossible à manipuler; les informaticiens, rechignant au travail sous prétexte que c'est trivial mais coûteux en temps; les membres du service administratif, réclamant de façon hebdomadaire des suivis d'activités chiffrés. Cette dernière exigence impose une gymnastique douloureuse car l'activité « réelle » est difficile à chiffrer puisqu'elle n'est qu'interactions, compromis et négociations.

Le contexte est complexe et d'actualité (intranet, communautés de pratique, échanges de savoir à distance, etc.) mais les tâches sont fastidieuses et non valorisées. Elles restent invisibles pour les personnes qui tiennent la parole publique (directeur du centre de recherche, clients et autorités administratives). Ces tâches quotidiennes, dévoreuses de temps ne sont visibles (trop visibles) que pour les protagonistes eux-mêmes qui les considèrent comme indignes. Les dirigeants et les administratifs ne comprennent pas la mauvaise humeur des services. Ils rajoutent alors des sanctions (gel de la notation annuelle), des contraintes (multiplications de comptes rendus écrits) ou diverses formes de mépris (paroles blessantes en réunions de direction ou simplement dans les couloirs). Un merci, un encouragement ou une gratification financière, comme forme de reconnaissance de ce sacrifice et l'assurance que cette surcharge est de courte durée auraient-ils changé le regard de chacun sur son travail en relation à celui des autres ?

\section{Visible, invisible ? Une question de gratitude, d'estime et de dignité}

L'analyse des terrains montre que la reconnaissance de l'individu au travail s'exprime dans des registres distincts selon la façon de concevoir l'invisibilité des tâches afférentes aux TIC. Dans le premier cas (industrie), l'invisibilité renvoie à l'appropriation achevée des dispositifs communicationnels dans les lieux de travail par des individus-utilisateurs valorisés et reconnus. L'usage des TIC s'inscrit 
ainsi dans la norme culturelle, est reconnu par la hiérarchie et se trouve légitimé par l'institution. Dans le second cas (centre de recherche), l'invisibilité renvoie à des activités fastidieuses et ingrates, obligatoires et peu honorables, malgré leur rôle indéniablement structurant. Une relation d'indifférence s'installe dans la routine professionnelle qui ne reconnaît pas dès lors la contribution sociale de l'individu.

Au sein des organisations, la question de l'estime est mobilisée en permanence mais elle est aussi imprégnée du contexte intime et sociétal. Qu'est-ce qui fait l'objet d'estime dans le cadre du travail ? Dans quelles mesures cette reconnaissance dans la sphère de la coopération sociale s'appuie-t-elle ou renforce-t-elle les formes de reconnaissance des deux autres sphères, publique et intime, le respect et la confiance ? Ces questions nous amènent à insister sur les marges de manœuvre et les registres de la justification que les individus mobilisent pour donner sens à leurs actions respectives.

Le degré de liberté dont bénéficient les individus au travail dépend non seulement de ce que nous avons nommé dispositifs communicationnels, mais aussi dispositifs normatifs. La définition des TIC en tant que dispositifs communicationnels nous permet d'élargir l'analyse au cadre plus large des pratiques sociales préexistantes tout en accordant une attention particulière à la situation (dans l'espace et dans le temps) et au contexte d'usage. Le dispositif communicationnel est pour l'utilisateur à la fois un cadre organisateur, un espace de contrainte (Foucault, 1975), mais aussi un espace de liberté, d'autonomie, de «bricolage» (de Certeau, 1990). D'autre part, le travail est un ensemble d'actions collectives encadrées par des rapports de prescriptions (Hatchuel, 2003) et de jugements. Ces rapports se matérialisent dans les instruments de gestion (Berry, 1983) que nous considérons comme des dispositifs normatifs. En effet, au même titre que les TIC, ils ne sont pas seulement techniques mais également chargés de pratiques sociales, ils cristallisent les rapports de force et offrent une marge de manœuvre. On peut les «bricoler» (Vacher, 2004). Dans le cas «industrie», les dispositifs communicationnels et normatifs (qui favorisent la responsabilité, l'autonomie, etc.) sont en phase avec l'action des individus leur donnant une certaine liberté et une ouverture vers la créativité. Dans le cas «centre de recherche» en revanche, ces dispositifs (suivis d'activités, applets, etc.) sont en décalage avec les actions habituelles des individus qui se referment sur leurs griefs. Nous voyons toutefois la simplicité d'améliorations éventuelles de ces dispositifs pour favoriser les encouragements et les gratifications. 
Dossier : Pour une approche communicationnelle de l'individu au travail

Pourquoi alors ces améliorations ne sont-elles pas mises en œuvre plus souvent? Nous proposons d'apporter un élément de réponse en insistant sur l'importance des registres de justification auxquels chacun se rattache. Ces registres renvoient à des principes considérés comme communs à tous les membres d'une cité donnée. Chaque individu de cette cité se sent digne dans ses capacités à agir pour le bien commun et grandit en légitimité chaque fois qu'il s'en rapproche (Boltanski, Thévenot, 1991). Au sein des organisations plusieurs cités cohabitent : la cité de l'inspiration (caractéristique du génie créatif ou du chercheur idéalisé) ; la cité domestique (univers lié à une hiérarchie d'ordre traditionnel), la cité de l'opinion (où seules comptent la renommée et la réputation); la cité civique (où prime le collectif au détriment du particulier), la cité marchande (celle des relations d'affaires où l'individu est mu par le désir de possession et la concurrence avec les autres) et la cité industrielle (dont l'idéal est le progrès et où sont reconnues la qualification, la performance et la productivité). Sur les terrains que nous avons étudiés, l'invisible ne produit pas les mêmes effets parce que les registres de justification auxquels font références les individus sont plus ou moins compatibles: les valeurs marchandes, industrielles et de l'opinion fonctionnent en harmonie avec celles de l'inspiration, de l'autorité et du civisme dans le cas « industrie ». Elles sont en porte à faux dans le cas « centre de recherche».

\section{Conclusion}

La problématique de la reconnaissance éclaire la communication des organisations sur plusieurs plans. Tout d'abord, la reconnaissance est un processus communicationnel en construction qui pose avec insistance les questions de visibilité et de légitimité des actions de l'individu au travail. Elle émerge au sein des contextes organisationnels dans les activités et les interactions entre individus et dispositifs normatifs et communicationnels. Pour échapper au mépris, à la méconnaissance et à l'invisibilité sociale, les salariés sont en quête d'une estime authentique, non seulement comme membres d'une «communauté » ou d'un groupe professionnel, mais surtout en tant qu'individus, autonomes et réflexifs. Dépendant des contraintes imposées par l'environnement, ils aspirent à des formes positives d'expressions empathiques. L'analyse des deux cas ici étudiés, fort différents de par leurs contextes, dispositifs et acteurs professionnels mobilisés, témoigne de l'importance grandissante de la problématique de la reconnaissance en entreprise à la lumière des nouveaux enjeux 
organisationnels ${ }^{60}$. Il pourrait être judicieux d'élargir cette problématique de la relation entre le visible et la reconnaissance au travail aux cadres sociétal et intime (Honneth, 2000, 2006). Ce qui est visible socialement est ce qui est mis en valeur par la société selon ses fondements culturels. La norme culturelle occidentale du capitalisme en réseau (Boltanski, Chiapello, 1999) ne pose-t-elle pas la question de la reconnaissance? Il ne suffit pas que celle-ci soit énoncée, il faut qu'elle s'exprime d'une manière visible, évidente, palpable, matérielle pour que l'individu puisse la saisir à travers le regard, la parole et l'action. C'est alors seulement qu'elle devient gratitude effective.

\section{Bibliographie}

ALMEIDA N. (d'), ANDONOVA Y., La communication des organisations. in : OLIVESI S. Coord. Sciences de l'Information et de la Communication. Objets, savoirs, discipline. Grenoble : PUG, 2006, pp. 129-143.

ALMEIDA N. (d'). Les promesses de la communication. Paris : PUF, 2001, $264 \mathrm{p}$.

ANDONOVA Y. L'enchevêtrement des techniques, des discours et des pratiques en milieu industriel. Contribution à une approche des usages des TIC. Thèse en SIC. Neuilly-sur-Seine : CELSA, Université Paris IV-Sorbonne, 2004, $500 \mathrm{p}$.

BERNARD F., JOULE R-V. Lien, sens et action: vers une communication engageante. in: Communication \& Organisation, 2004, n²4, pp. 333-345.

BERRY M. Une technologie invisible? L'impact des instruments de gestion sur l'évolution des systèmes humains. Paris: Centre de Recherche en Gestion, École Polytechnique, 1983, 93 p.

BOLTANSKI L. THÉVENOT L. De la justification. Les économies de la grandeur. Paris : Gallimard, 1991, 483 p.

BOLTANSKI L., CHIAPELLO E. Le nouvel esprit du capitalisme. Paris : Gallimard, 1999, 843 p.

BORZEIX A., FRAENKEL B. Langage et Travail. Communication, cognition, action. Paris : CNRS Éditions, 2005, 379 p.

\footnotetext{
${ }^{60}$ Les résultats de ces études de cas ne nous permettent pas de généraliser la portée de l'analyse, mais de discerner des tendances significatives.
} 
Dossier : Pour une approche communicationnelle de l'individu au travail

BOUZON A. Communiquer dans l'incertain : La communication dans les processus de conception innovante à "risques maîtrisés». HDR en SIC. Toulouse : Université Paul Sabatier, 2002, 345 p.

CERTEAU M. (de). L'invention du quotidien. 1. arts de faire. Paris : Gallimard, 1990, 347 p.

FLORIS B. Parlons-nous d'organisation en «chair et en os »? in : BOUZON A. Coord. La communication organisationnelle en débat: champs, concepts, perspectives. Paris : L'Harmattan, 2006, pp. 123129.

FOUCAULT M. Surveiller et punir. Paris : Gallimard, 1975, 328 p.

GIROUX N. Le nouage des savoirs en organisation. in : BOUZON A. Coord. La communication organisationnelle en débat: champs, concepts, perspectives. Paris : L'Harmattan, 2006, pp. 159-189.

GIROUX N., MARROQUIN L. L'approche narrative des organisations. in : Revue française de gestion, 2005, $\mathrm{n}^{\circ} 159$, pp. 15-42.

GIROUX N. La gestion discursive des paradoxes de l'identité. in : DOSTALER I., LAROCHE H., BOIRAL O. Coord. Perspectives en Management stratégique. Paris : EMS, vol.8, 2002, pp. 133-163.

GLASER B., STRAUSS A. The Discovery of Grounded Theory: Strategies for Qualitative Research. Chicago: Aldine de Gruyter, 1967, $271 \mathrm{p}$.

GOFFMAN E. Les cadres de l'expérience. Paris : Éditions de minuit, 1991, $573 \mathrm{p}$.

GRAMACCIA G. Les actes de langage dans les organisations. Paris : L'Harmattan, 2001, 290 p.

GUYOT B. Éléments pour une approche informationnelle dans les organisations. in : Sciences de la société, 2004, nº63, pp. 11-26.

HABERMAS J. Théorie de l'agir communicationnel. Paris : Fayard, 1987, $480 \mathrm{p}$.

HATCHUEL A. La théorie de la régulation est-elle une théorie de l'action collective? in: DE TERSSAC G. Coord. La théorie de la régulation de Jean-Daniel Reynaud. Paris: La Découverte, 2003, pp. 289-302.

HONNETH A. La Lutte pour la reconnaissance. Paris ; Ed. du Cerf, 2000, $233 \mathrm{p}$. 
HONNETH A. La société du mépris. Vers une nouvelle Théorie critique. Paris : La Découverte, 2006, 349 p.

HUTCHINS E. Cognition in the Wild. Cambridge/Londres: MIT Press, 1995, $381 \mathrm{p}$.

LACOSTE M. L'espace du langage. De l'accomplissement du travail à son organisation. in : Sciences de la Société, 2000, n50/51, pp. 197215.

MAUSS M. Essai sur le don. Forme et raison de l'échange dans les sociétés archaïques. in : L'Année Sociologique, seconde série, 1, 1925.

MEDA D. Le Travail. Paris : PUF, 2004, 128 p.

OLIVESI S. La communication au travail. Une critique des nouvelles formes de pouvoir dans les entreprises. Grenoble : PUG, 2002, 164 p.

POCHE F. Une politique de la fragilité. Éthique, dignité et luttes sociales. Paris : Ed. du Cerf, 2004, 257 p.

RICEUR P. Parcours de la reconnaissance. Paris: Stock, 2004, $386 \mathrm{p}$.

SUCHMAN L. Plans and situated actions, the problem of humanmachine communication. New York: Cambridge University Press, 1987, $203 \mathrm{p}$.

TAYLOR J.R., COOREN F., GIROUX, N., ROBICHAUD D. The communicational basis of organization : Between the conversation and the text. in : Communication Theory, 1996, 6 (1), pp. 1-39.

VACHER B. Du bricolage informationnel à la litote organisationnelle. Ou comment considérer le bricolage au niveau stratégique? in: Sciences de la Société, 2004, n63, pp. 133-150.

VACHER B. L'invisible et structurante matérialité de l'information et de la communication organisationnelles: une grille de lecture. in: Communication \& Organisation, 2006, $\mathrm{n}^{\circ} 30$, pp. 230-249.

VACHER B. Combiner Science de l'Information et de la Communication et Sciences de Gestion pour étudier l'action collective. in : actes du XVI ème congrès de la SFSIC, Les sciences de l'information et de la communication : affirmation et pluralité, 11-13 juin 2008, Compiègne.

WINKIN Y. Anthropologie de la communication. De la théorie au terrain. Bruxelles : Éditions De Boeck Université, 1996, 235 p. 